\title{
An Integrative View of Cisplatin Uptake by Kidney and Renal Toxicity Amr Ahmed EL-Arabey*
}

Pharmacology and Toxicology Department, Faculty of Pharmacy, Al-Azhar University, Nasr City, Cairo, Egypt

*Corresponding author: Amr Ahmed EL-Arabey, Pharmacology and Toxicology Department, Faculty of Pharmacy, Al-Azhar University, Nasr City, Cairo, Egypt, Tel: +39 0187533213; E-mail: ph.amrcapa@gmail.com

Received date: Feb 05, 2018; Accepted date: Feb 07, 2018; Published date: Feb 12, 2018

Copyright: (C) 2018 EL-Arabey AA. This is an open-access article distributed under the terms of the Creative Commons Attribution License, which permits unrestricted use, distribution, and reproduction in any medium, provided the original author and source are credited.

\section{Editorial}

Cisplatin (cis-diamminedichloroplatinum II) (CDDP) is an inorganic platinum-based chemotherapeutic agent that is widely used in the treatment of a variety of solid organ cancers. The main dose limiting side effect of CDDP is nephrotoxicity; after a single dose of CDDP $\left(50-100 \mathrm{mg} / \mathrm{m}^{2}\right)$, approximately one-third of the patients develop nephrotoxicity. Yet, in spite of intense efforts over the ensuing decades to find less toxic but equally effective alternatives, CDDP continues to be widely prescribed as a standard component of treatment regimens for head and neck cancers, small-cell and nonsmall cell lung cancer, testicular cancer, ovarian, cervical cancer, bladder cancer, melanoma and others. The CDDP chief dose-limiting nephrotoxic side effect requires a reduction of the dose or discontinuation of treatment $[1,2]$.

Amifostine is used in reducing progressive nephrotoxicity of repeated CDDP dosing in patients with advanced ovarian cancer and approved by FDA. The protective effects of amifostine derived by providing a thiol group to normal versus malignant cells [3]. Unfortunately, renal toxicity still occurs even with amifostine. Hence, this has prompted the development of more effective preventive strategies. Renal poisoning from CDDP originates from the uptake and stimulation of platinum at proximal tubule. Therefore, manipulator which differentially reduce CDDP uptake by the kidney relative to tumor cells, should reduce nephrotoxicity without reducing anti-tumor responses [4].

Every cell has a membrane that separates its interior from the external environment, for this reason vital processes like entry of all essential nutrients into the cytoplasmic compartment, distribution of cellular products into and beyond the cellular membrane, handling of toxic materials and waste products are necessarily mediated by a variety of different transport proteins [5]. Thus, transporters influences human physiology and pathophysiology and are critical key players of therapeutic response to drugs. Drug transporters regulate absorption, distribution and metabolism as well as drugs excretion because of their higher expression in the kidney, liver, intestine and endothelial barriers [6]. A number of important human drug transporters have been distinguished that are distributed at the apical or basal aspect of the epithelial cells in various tissues [7]. Transporters mediated cellular uptake or outflow of drugs; therefore, they can mediate drug efficacy, resistance and undesired toxic effects, as confirmed for example for CDDP [6].

Organic cation transporters (OCTs) have been implicated in CDDP uptake. The CDDP-induced tubular cell injury may be related to basolateral OCT. OCT1, OCT2 and OCT3 are three isoforms of OCTs which expressed in the basolateral part of renal proximal tubules [8]. Furthermore, it has been deduced that OCT2 (Figure 1A) in the kidney and that CDDP uptake was increased by over expression of
OCT2 in HEK293 cells and associated with increased cellular sensitivity to CDDP toxicity [9]. OCT2 is most fundamentally expressed in the kidney and is also expressed in the small intestine, lung, placenta, skin, brain and choroid plexus [10]. Interestingly, OCT2 mediates uptake from the blood to the proximal tubular cells during the renal secretion of organic cations. Thus, OCT2 plays crucial role on the pharmacological, pharmacokinetic and toxicological features of therapeutics. In addition, CDDP nephrotoxicity is gender dependent and previously, I suggested these differences may be related to CDDP uptake by OCT2 due to the markedly higher renal expression of OCT2 in male than female rats $[11,12]$. Practically, a study by Sprowl et al. in 2014 elucidated that OCT1/2 (-/-) mice were not completely protected from CDDP-induced tubular necrosis, while the loss of p53 in OCT1/2 (-/-) mice conferred complete protection [13].

Organic anion transporter 1 (OAT1) (Figure 1B) and OAT3 (Figure 1C) in the kidney proximal tubules and play important roles in the elimination of harmful endogenous compounds and xenobiotics from the body.

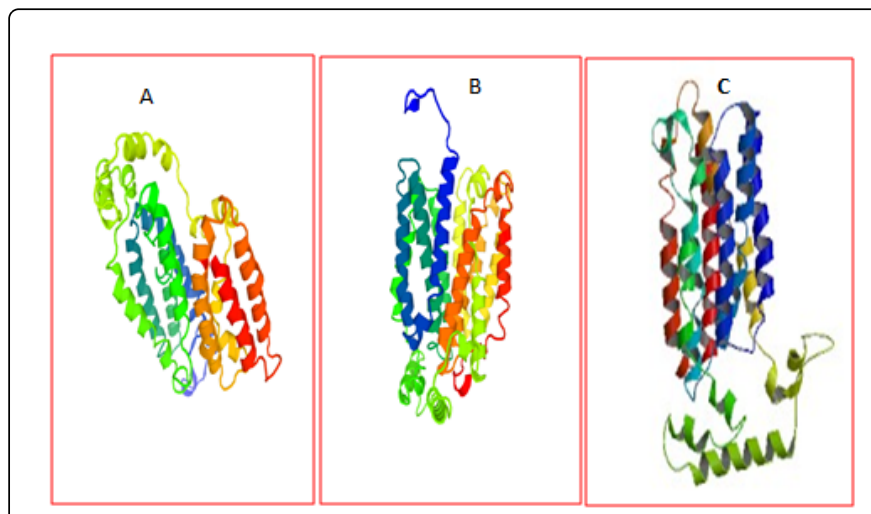

Figure 1: 2D structure of (A) hOCT2 (residue range: 126-499). (B) hOAT1 (residue range: 121-515). (C) hOAT3 (residue range: 121-407).

Recently, work by $\mathrm{Hu}$ et al. revealed that OAT1/OAT3 is a novel metabolite a mercapturic acid CDDP uptake pathway independent of the OCT2 pathway of CDDP nephrotoxicity and that act upstream of p53 [14]. Collectively, OCT2, OAT1 and OAT3 are critical transporters in CDDP-induced kidney injury. Moreover, further work on the regulation of these transporters could add novel candidate compounds to improve the current state of CDDP therapeutics and offer more alternatives choice for clinicians and patients to promote longer survival and better quality of life. 
Citation: EL-Arabey AA (2018) An Integrative View of Cisplatin Uptake by Kidney and Renal Toxicity. J Kidney 4: e110. doi:

\section{References}

1. EL-Arabey AA, Abd-Allah AR (2015) Antidepressants as a new approach for protective interventions of cisplatin-induced nephrotoxicity. J Kidney 1: 102 .

2. El-Arabey AA (2016) Dual function of OCT2 and MATE1 in cisplatin induced nephrotoxicity. Pharmacol Res 119: 493.

3. Hensley ML, Hagerty KL, Kewalramani T, Green DM, Meropol NJ, et al. (2009) American Society of Clinical Oncology 2008 clinical practice guideline update: Use of chemotherapy and radiation therapy protectants. J Clin Oncol 27: 127-145.

4. Saier MH Jr (2000) A functional-phylogenetic classification system for transmembrane solute transporters. Microbiol Mol Biol Rev 64: 354-411.

5. Ciarimboli G (2011) Role of organic cation transporters in drug-induced toxicity. Expert Opin Drug Metab Toxicol 7: 159-174.

6. Wright SH (2005) Role of organic cation transporters in the renal handling of therapeutic agents and xenobiotics. Toxicol App Pharmacol 204: 309-319.

7. Yonezawa A, Masuda S, Nishihara K, Yano I, Katsura T, et al. (2005) Association between tubular toxicity of cisplatin and expression of organic cation transporter rOCT2 (Slc22a2) in the rat. Biochem Pharmacol 70: 1823-1831.
8. EL Arabey AA, Salama AS (2015) Are mice and rats good experimental models to explore novel compounds against cisplatin induced nephrotoxicity? Int J Nephrol Kid Failure 1.

9. Motohashi H, Sakurai Y, Saito H, Masuda S, Urakami Y, et al. (2002) Gene expression levels and immunolocalization of organic ion transporters in the human kidney. J Am Soc Nephrol 13: 866-874.

10. Ciarimboli G, Ludwig T, Lang D, Pavenstadt H, Koepsell H, et al. (2005) Cisplatin nephrotoxicity is critically mediated via the human organic cation transporter 2. Am J Pathol 167: 1477-1484.

11. El-Arabey AA (2015) Gender difference in Cisplatin-induced nephrotoxicity in a rat model. Nephrourol Mon 7: e23595.

12. El-Arabey AA (2015) Sex and age differences related to renal OCT2 gene expression in cisplatin-induced nephrotoxicity. Iran J Kidney Dis 9: 335-336.

13. Sprowl JA, Lancaster CS, Pabla N, Hermann E, Kosloske AM, et al. (2014) Cisplatin-induced renal injury is independently mediated by OCT2 and p53. Clin Cancer Res 20: 4026-4035.

14. Hu S, Leblanc AF, Gibson AA, Hong KW, Kim JY, et al. (2017) Identification of OAT1/OAT3 as contributors to cisplatin toxicity. Clin Transl Sci 10: 412-420. 